(2) Open Access Full Text Article

\title{
Monozygotic twins with polypoidal choroidal vasuculopathy
}

This article was published in the following Dove Press journal:

Clinical Ophthalmology

10 July 2010

Number of times this article has been viewed

\author{
Shigeki Machida' \\ Tomomi Takahashi' \\ Norimoto Gotoh ${ }^{2}$ \\ Nagahisa Yoshimura ${ }^{2}$ \\ Takamitsu Fujiwara' \\ Dajiro Kurosaka' \\ 'Department of Ophthalmology, \\ Iwate Medical University School \\ of Medicine, ${ }^{2}$ Department of \\ Ophthalmology and Visual Sciences, \\ Kyoto University Graduate School \\ of Medicine
}

Correspondence: Shigeki Machida Department of Ophthalmology, Iwate Medical University School of Medicine, 19-I Uchimaru Morioka Iwate 020-8505, Japan

$\mathrm{Tel}+81196515111$

Fax +8I 196532864

Email smachida@iwate-med.ac.jp
Purpose: To present the first findings in the set of monozygotic twins with polypoidal choroidopathy (PCV).

Methods: Sixty two-year old monozygotic twin sisters were studied. The concordances and discordances of the clinical features of the twins were determined. Genomic DNA was extracted and genotyped for three established PCV risk-associated single nucleotide polymorphisms, viz CFH I62V, CFH Y402H, and ARMS A69S.

Results: Both patients had hemorrhagic pigment epithelial detachments with orange lesions beneath the retinal pigment epithelium. Indocyanine green angiography showed pathognomonic choroidal vascular networks with polypoidal structures uniocularly in one twin and binocularly in the other twin. Both twins were treated with photodynamic therapy, retinal photocoagulation, and anti-vascular endothelial growth factor therapy, but both showed limited response to all the treatments, with recurrent exudative lesions with enlarged vascular network, and poor visual outcome. Genetic analyses showed that both sisters had homozygous risk alleles for ARMS2 A69S, and one risk allele each of CFH I62V and CFH Y402H.

Conclusions: We present the first findings in a set of monozygotic twins with typical PCV under long-term observation. The concordances in disease progression and response to treatment between the twins indicate that these genetic factors most likely played important roles in determining the clinical manifestations.

Keywords: polypoidal choroidal vasculopathy, PCV, CFH, ARMS2, monozygotic twins

\section{Introduction}

Polypoidal choroidal vasculopathy (PCV) is a sight-threatening eye disease of older individuals, and is characterized by dilatations at the ends of abnormal blood vessels in the inner choroidal vascular network. ${ }^{1,2} \mathrm{PCV}$ is a distinct form of age-related macular degeneration (AMD). Recent studies have found similar risk factors in PCV and AMD. ${ }^{3,4}$ As an environmental factor, smoking is not only an established risk for AMD, but also for PCV. ${ }^{5}$ As genetic factors, single nucleotide polymorphisms (SNPs) of the complement factor $\mathrm{H}(\mathrm{CFH})$ gene $^{6-9}$ and the age-related maculopathy susceptibility

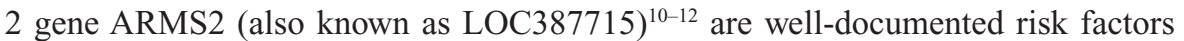
for AMD. ${ }^{13}$ These risk polymorphisms are also associated with the development and progression of $\mathrm{PCV}^{4,14-17}$

Monozygotic and dizygotic twins are ideal models in which to study the role of genes in determining the phenotype of diseases. A high concordance of the phenotypes in monozygotic twins would strongly suggest that genetics played a larger role in their development than environmental factors. Klein et $\mathrm{al}^{18}$ demonstrated a high concordance of phenotype in nine pairs of monozygotic twins with AMD. Seddon et al ${ }^{19}$ demonstrated the 
role of heredity in the development and progression of AMD using the largest cohort ever examined (840 male twins).

We shall present our findings in a set of monozygotic twins who had the characteristic features of PCV, where genetic analyses showed that they both carried polymorphisms of the CFH and ARMS2 genes. To the best of our knowledge, this is the first presentation of findings in a set of monozygotic twins with PCV under long-term observation.

\section{Patients and methods}

\section{Patients}

We studied a set of monozygotic twin sisters who were referred to the Retina Unit of Iwate Medical University Hospital. Case 1 was followed from October 2004 to January 2010. Case 2 was followed from February 2004 from to December 2009. Although Cases 1 and 2 were monozygotic twins, they had grown up in different families in different cities since birth, due to personal reasons.

\section{Methods}

Both patients received comprehensive ophthalmological examination, including Snellen visual acuity tests, indirect ophthalmoscopy, and slit-lamp examination at every visit. In addition, color fundus photographs, fluorescein angiography (FAG), and indocyanine green angiography (ICGA) were recorded before and after treatments.

After a full explanation of the nature of the study and the procedures to be used, an informed consent was obtained from both sisters. Genetic analysis was conducted in accordance with the methods approved by the Institutional Review Board/Ethics Committee of the Iwate Medical University and Kyoto University. Genomic DNA isolations were prepared with QuickGene-610L ${ }^{\circledR}$ (Fujifilm, Minato, Tokyo, Japan) from the patients' peripheral blood. Genotyping for the three PCV-associated SNPs [rs10490924 (A69S) of the ARMS2 gene, and rs800292 (I62V) and rs1061170 (Y402H) of the CFH gene] was performed with Taqman ${ }^{\circledR}$ SNP assays (Applied Biosystems, Foster City, CA. USA) using NCBI dbSNP build 128 with the ABI PRISM ${ }^{\circledR} 7900$ HT (Applied Biosystems) according to the manufacturer's instructions.

\section{Results \\ Case reports}

\section{Case I}

Case 1 was 62 years old at the time of the initial examination and was the first of the twins born. She visited us in October 2004 with a one-month history of metamorphopsia and decreased vision in her right eye. She had no medical history and had never smoked. She weighed $58 \mathrm{~kg}$ and her body mass index (BMI) was 24.1. Her best corrected visual acuity (BCVA) was 25/20 in both eyes.

A hemorrhagic pigment epithelial detachment (PED) involving the fovea with subretinal hemorrhage inferior to the lower vascular arcade was detected in the right eye (Figure 1A). Two round orange lesions were seen beneath the retinal pigment epithelium (RPE) in the supero-temporal region of the macula (Figure 1A, arrows). Fluorescein angiography (FA) showed dye pooling in the PED (Figure 1B). ICGA showed a choroidal vascular network and two polypoidal lesions at the edge of the PED (Figure 1C). Dye leakage from the polypoidal lesions was seen in the late phase of ICGA (Figure 1D).

The greatest linear dimension (GLD) of the lesion was $3,580 \mu \mathrm{m}$. The choroidal vascular network and polypoidal lesions were treated with photodynamic therapy (PDT) with verteporphin (Visdyne ${ }^{\circledR}$, QLT Ophthalmics, Menlo Park, CA, USA). The hemorrhagic PED disappeared 3 months later with resolution of the metamorphopsia and retention of vision (Figure 1E). ICGA demonstrated closure of the polypoidal lesions (Figure 1F).

In March 2008, 3 years and 5 months after the initial PDT, a PED and subretinal hemorrhage involving the macula developed, with a deterioration of the BCVA to 20/100 (Figure 2A). FA showed pooling of dye beneath the PED and leakage of dye in the macular region, suggesting the presence of an occult choroidal neovascularization (Figure 2B). In the early phase of ICGA, abnormal choroidal vessels were seen to extend to the macula (Figure 2C, arrows). Extensive dye leakage from the polypoidal structures and diffuse staining of the macula were seen in the late phase ICGA (Figure 2D).

Laser photocoagulation was applied to the polypoidal lesions at the edge of the PED, leading to the disappearance of the PED. However, dye leakage from the macular lesion further deteriorated the vision postoperatively. We injected bevacizumab intravitreally, which was followed by PDT. A GLD of 5,500 $\mu \mathrm{m}$ was observed 7 days later. Although the exudative changes subsided, fibrovascular tissue beneath the macula kept the BCVA at 20/400 (Figure 2E and 2F). A serous retinal detachment associated with subretinal fibrosis was observed at the final visit in January 2010.

No abnormal signs were observed in the left eye funduscopically and angiographically throughout the follow-up examinations.

\section{Case 2}

Case 2 was the second-born twin, who visited us in February 2004 complaining of decreased vision in the 


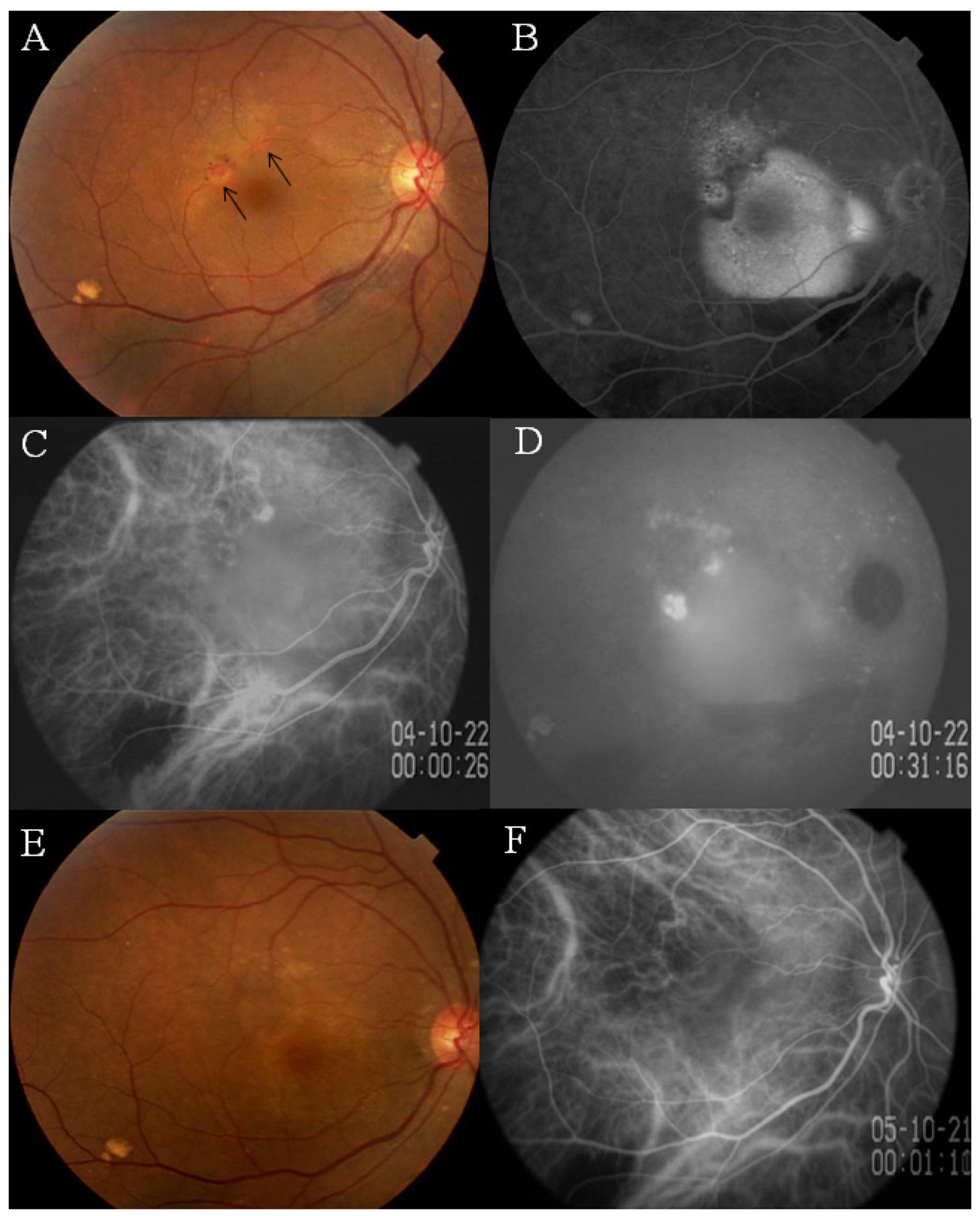

Figure I Case I. Findings in a 62-year-old monozygotic twin with polypoidal choroidal vasculopathy in her right eye.

Notes: A) Fundus photograph of right eye shows a hemorrhagic pigment epithelial detachment (PED) associated with an orange lesion beneath the retinal pigment epithelium (RPE) (arrows). B) Fluorescein angiogram shows dye pooling beneath the RPE. C) Early phase indocyanine green angiogram (ICGA) showing a network of vessels and polypoidal structures. D) Late phase ICGA showing intense dye leakage from the polypoidal lesions. E) Three months after photodynamic therapy, the PED cannot be seen. F) ICGA showing closure of the polypoidal lesions.

left eye. She had never smoked, and weighed $62 \mathrm{~kg}$. Her BMI was 25.8. She had a history of a hemorrhagic PED in the right eye that had been treated with photocoagulation when she was 57-years-old (Figure 3A). Her BCVA was 40/50 OD and 20/60 OS. Although she had not been treated with medications, serial measurements of blood pressure revealed that she had systemic hypertension.

The fundus of the right eye showed chorioretinal atrophy induced by laser photocoagulation and RPE atrophy (Figure 3B). The left eye had a hemorrhagic PED involving 


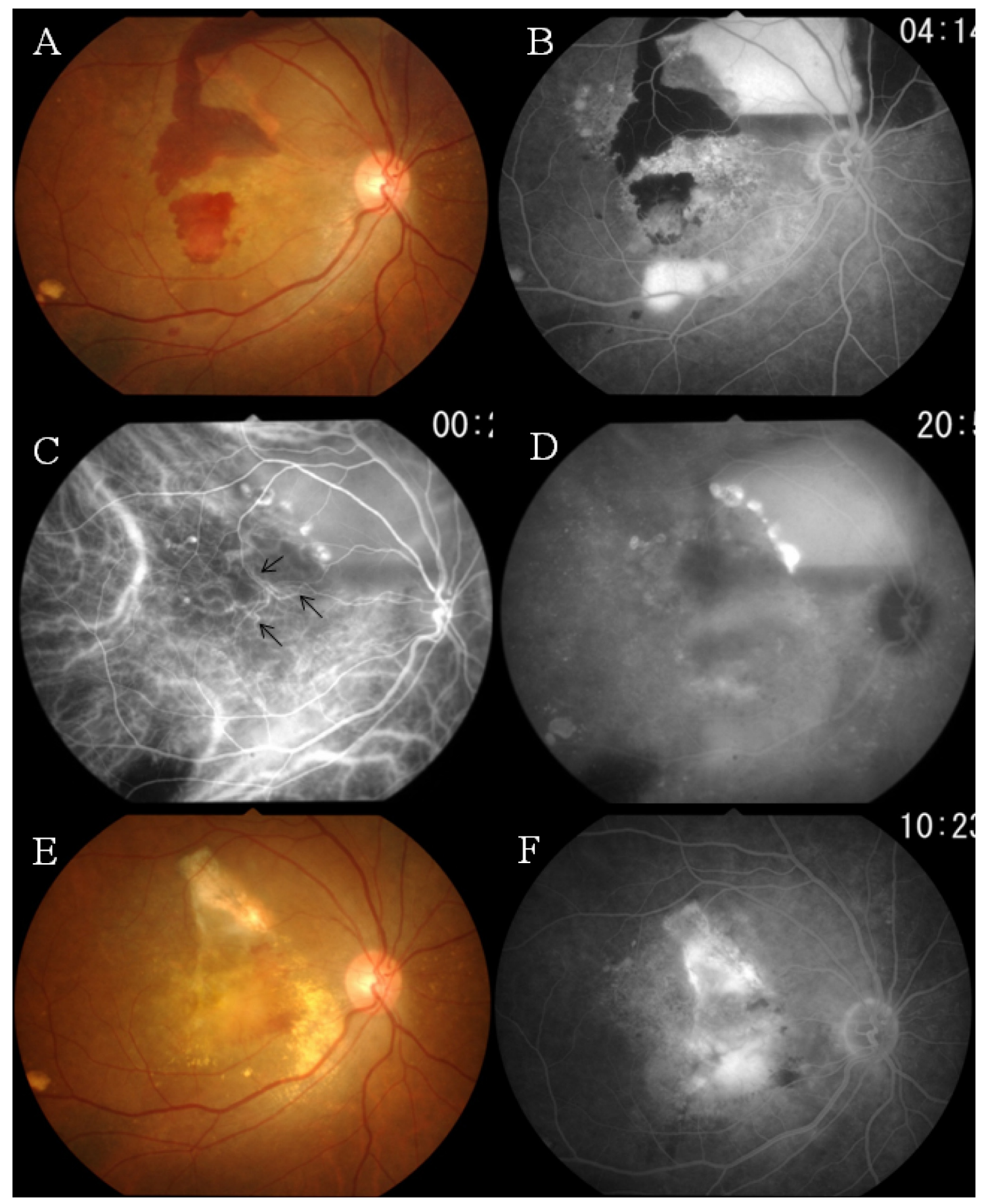

Figure 2 Case I. Three years and five months after the initial photodynamic therapy (PDT).

Notes: A) Hemorrhagic pigment epithelial detachment (PED) and subretinal hemorrhage have recurred. B) Fluorescein angiogram shows pooling of dye beneath the PED and leakage of dye in the macular region. C) Indocyanine green angiogram (ICGA) delineates the polypoidal lesions and abnormal choroidal vessels that have extended from the treated site (arrows). D) Late phase of ICGA: extensive dye leakage from the polypoidal lesions and diffuse staining involving the macula. E) After photocoagulation applied to the polypoidal lesions and PDT combined with an intravitreal injection of bevacizumab: the exudative changes have subsided, leaving a subretinal fibrovascular tissue. F) Dye leakage from the fibrovascular tissue is still present.

the fovea which was surrounded by exudative retinal detachment (Figure 3C). FA confirmed the PED as a hyperfluorescent area (Figure 3D). Early phase ICGA showed two polypoidal structures and a choroidal network of vessels with dye leakage in the late phase (Figures $3 \mathrm{E}$ and $3 \mathrm{~F}$ ).
She was treated with direct irradiation at 20 Gy to the posterior pole of the eye (because PDT was not available in Japan at that time). Three years after the irradiation, in March 2006, the patient was referred to us complaining of further deterioration of vision (to 20/200) in her left eye. 


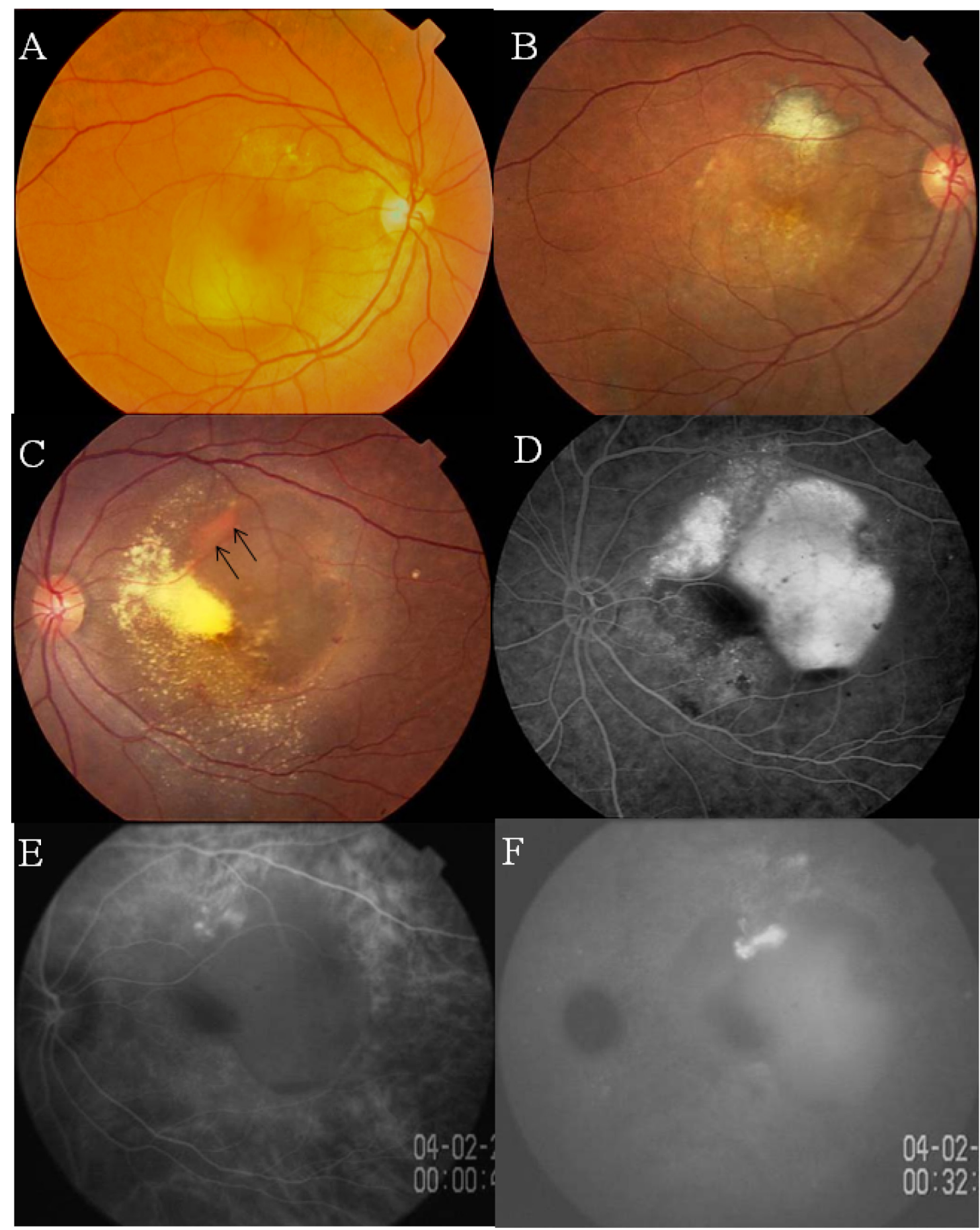

Figure 3 Case 2. Twin sister of Case I with polypoidal choroidal vasculopathy in both eyes.

Notes: A, B) Fundus photographs of right eye before (A) and after (B) laser photocoagulation at the age of 57 years. C) Photograph of left fundus showing an orange lesion at the margin of a hemorrhagic PED (arrows). D) Fluorescein angiogram showing leakage of dye beneath the PED. E, F) Indocyanine green angiogram (ICGA) of right eye showing polypoidal lesions at the margin of the PED (E) with late dye leakage (F).

Examination showed an enlargement of the hemorrhagic PED, which was confirmed by FAG (Figure 4A and 4B). ICGA revealed newly-developed polypoidal structures and a choroidal network of vessels (Figure 4C). ICGA-guided PDT was applied to the polypoidal structures and vascular network with two laser spots (GLD: 2,600 $\mu \mathrm{m}$ and 3,600 $\mu \mathrm{m}$ ), which resulted in resolution of the exudative lesions and recovery of BCVA to 20/125 (Figure 4D).

In January 2007, 7 months after the initial PDT, exudative changes recurred, with reopening and further development of the polypoidal vascular lesions. These changes required additional treatments, including a second PDT combined with an 


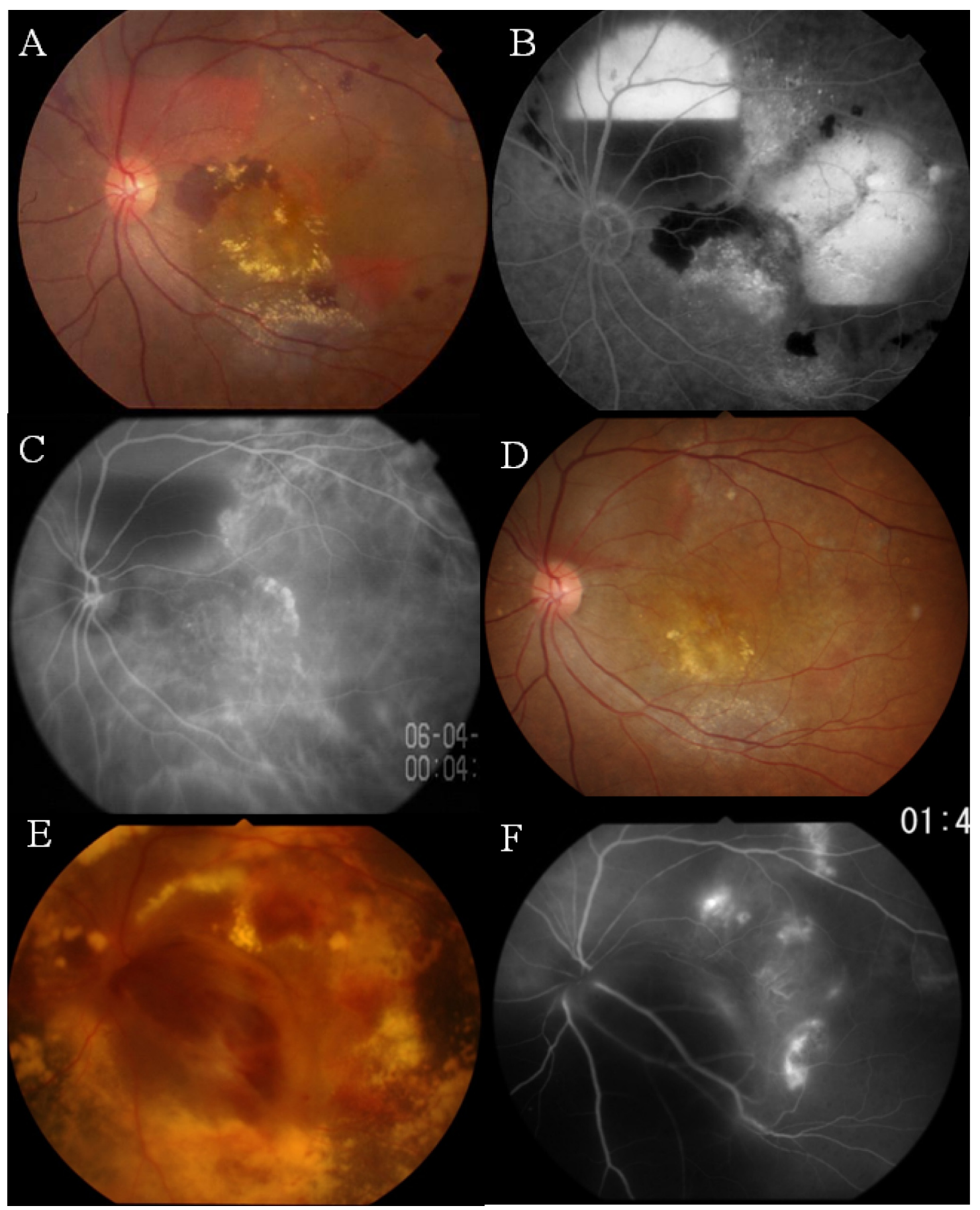

Figure 4 Case 2. Three years after the direct irradiation.

Notes: A, B) Fundus photograph (A) showing the recurrence of hemorrhagic PEDs which can be seen as leakage of fluorescein in the fluorescein angiogram (B). C) Indocyanine green angiogram showing newly-developed polypoidal structures and network vessels. D) After PDT: the exudative changes have subsided. E, F) Fundus photograph (E) seven months after PDT, showing the massive subretinal hemorrhage that has developed, with further extension of the polypoidal lesions and network vessels $(\mathbf{F})$.

intravitreal injection of bevacizumab and repeated injections of rabnizumab into the vitreous humor, which did not have favorable effects on the exudative changes. The most recent BCVA was 20/800. The vascular network and polypoidal structures had increased, with massive subretinal deposits and hemorrhagic PEDs (Figure 4E and 4F) in December 2009.

\section{Genetic Variants}

The genetic polymorphisms of Cases 1 and 2 are shown in Table 1. The twins had heterozygous gene polymorphisms of CFH (GA and CT for I62V and Y402H respectively). In addition, they had homozygous risk alleles for the ARMS2 polymorphisms (TT for A69S). 
Table I Genetic polymorphisms of CFH and ARMS2 (LOC 387715)

\begin{tabular}{llll}
\hline & Case I & Case 2 & Risk allele \\
\hline CFHI62V & GA & GA & G \\
CFHY402H & CT & CT & C \\
ARMS2 (LOC3877I5) A698 & TT & TT & T \\
\hline
\end{tabular}

\section{Discussion}

We have presented the clinical and genetic findings for a set of monozygotic twin sisters with PCV. They had high concordances in their clinical characteristics, including funduscopic and angiographic findings, responses to treatments, and visual prognoses. In addition, they had identical PCV-susceptible allele distributions.

\section{Validity of diagnosis}

Both cases had pathognomonic manifestations of PCV, including polypoidal structures and choroidal vascular networks. In addition, both had orange lesions beneath the RPE and recurrent hemorrhagic PEDs. These clinical findings were concordant with earlier descriptions of PCV. ${ }^{1,2}$ In the past decade, ICGA has been established as a cardinal diagnostic tool for PCV. ${ }^{2}$ With ICGA, our cases showed polypoidal structures and choroidal vascular networks that are characteristic of PCV.

The prevalence of PCV is high in the Asian population. It accounts for $24 \%-55 \%$ of all AMD patients. ${ }^{20-22}$ To make correct diagnoses of PCV in the clinics of Asia, the Japanese Study Group of Polypoidal Choroidal Vasculopathy published the diagnostic criteria for PCV. ${ }^{23}$ The fundus of the twins showed protruded orange lesions beneath the RPE and polypoidal vascular lesions detected by ICGA, which meet the diagnostic criteria of typical PCV.

\section{Concordances and discordances in clinical features}

Both cases had extarafoveal vascular lesions accompanied by large hemorrhagic PEDs at an early stage. With time, the vascular lesions enlarged and extended to the macular region. In addition to the typical manifestations of PCV, our cases shared similar clinical features such as size of lesion, response to treatment, and visual outcome. At initial visits, the responsible lesions detected by ICGA were relatively small and treatable by PDT. However, the vascular lesions progressively grew, and finally the GLD reached $>5,000 \mu \mathrm{m}$. Both twins were treated with PDT, which resulted in a transient resolution of the exudative changes and restoration of vision. However, both cases had recurrences of the exudative changes, which led to further deterioration of vision and the need for additional treatment. These recurrences greatly depressed the foveal function and finally reduced the vision to low levels.

We also found some differences in the clinical features between the sisters. The ocular lesion was unilateral in Case 1, while it was bilateral in Case 2. The ocular symptoms started at age 62 years in Case 1, while they started at 57 years in Case 2. Earlier publications have reported that environmental factors such as smoking, ${ }^{24}$ systemic hypertension, ${ }^{25}$ obesity, ${ }^{26}$ and nutritional factors contribute to the development of AMD. Although the sisters are monozygotic twins, they grew up since birth in different households and in different cities, suggesting that environmental factors were somewhat different.

The BMI was slightly higher in Case 2 than in Case 1. In addition, Case 2 had systemic hypertension. It is possible that environmental factors were responsible for the phenotypic differences between the sisters.

\section{Distribution of PCV-risk polymorphisms}

We found three genetic polymorphism distributions in two genes: ARMS2 A69S and CFH I62V and $\mathrm{Y} 402 \mathrm{H}$. CFH is an essential regulatory protein that plays a critical role in the homeostasis of the complement system. ${ }^{27}$ The CFH gene variants could result in uncontrolled complement activation, which is consistent with earlier reports which suggest alterations of the immune system as the pathophysiology of AMD. ${ }^{28}$ Although the function of ARMS2 has not been determined, it has been shown that ARMS2 is located in the mitochondrial outer membrane. This would suggest that the ARMS2 gene variant may affect the mitochondrial function of photoreceptors. ${ }^{29}$

Recent studies have demonstrated significant associations between PCV and the genetic variants of CFH and ARMS2, even in the Japanese population. ${ }^{4,14-17}$ Furthermore, a combination of variants of the CFH and ARMS2 genes have been shown to enhance the risk of developing PCV. ${ }^{4}$ Mori et $\mathrm{al}^{4}$ reported that the joint odds ratio for the combination of genotypes TT for ARMS2 A69S and CT for CFH Y402H (the same combination seen in our twins) was 6.65. In addition, the homozygous risk alleles for the ARMS2 gene were reported to be highly associated with massive subretinal hemorrhage, vitreous hemorrhage, and larger lesion size in Japanese patients with PCV. ${ }^{14,17,30}$ Therefore, our cases had high-risk alleles for the development, progression, and advancement of PCV. In fact, our cases had repeated recurrences and unfavorable visual outcomes despite treatment. 


\section{Conclusions}

We have reported the phenotypes and genotypes of a set of monozygotic twins with typical clinical features of PCV. The resemblances in the progression of the disease and response to treatment between the twins indicate that genetic factors played important roles in determining their clinical manifestations.

\section{Disclosure}

The authors report no conflicts of interest in this work.

\section{References}

1. Yannuzzi LA, Sorenson J, Spaide RF, et al. Idiopathic polypoidal choroidopathy (IPCV). Retina. 1990;10:1-8.

2. Spaide RF, Yannuzzi LA, Slakter JS, et al. Indocyanine green videoangiography of idiopathic polypoidal choroidal vasculopathy. Retina. 1995; 15:100-110.

3. Laude A, Cackett PD, Vithana EN, et al. Polypoidal choroidal vasculopathy and neovascular age-related macular degeneration: same or different disease? Prog Retin Eye Res. 2010;29:19-29.

4. Mori K, Horie-Inoue K, Gehbach PL, et al. Phenotype and genotype characteristics of age-related macular degeneration in a Japanese population. Ophthalmology. 2010, Epub ahead of print.

5. Kikuchi M, Nakamura M, Ishikawa K, et al. Elevated C-reactive protein levels in patients with polypoidal choroidal vasculopathy and patients with neovascular age-related macular degeneration. Ophthalmology. 2007;114:1722-1727.

6. Klein RJ, Zeiss C, Chew E, et al. Complement factor $\mathrm{H}$ polymorphism in age-related macular degeneration. Science. 2005;308:385-389.

7. Jonathan HL, Hauser MA, Schmidt S, et al. Complement factor $\mathrm{H}$ variant increases the risk of age-related macular degeneration. Science. 2005;308:419-421.

8. Edwards AO, Ritter R III, Abel KJ, et al. Complement factor H polymorphism and age-related macular degeneration. Science. 2005;308: 421-424.

9. Hageman GS, Anderson DH, Johnson LV, et al. A common haplotype in the complement regulatory gene factor $\mathrm{H}(\mathrm{HF} 1 / \mathrm{CFH})$ predisposes individuals to age-related macular degeneration. Proc Natl Acad Sci U SA. 2005;102:7227-7232.

10. Rivera A, Fisher SA, Fritsche LG, et al. Hypothetical LOC387715 is a second major susceptibility gene for age-related macular degeneration, contributing independently of complement factor $\mathrm{H}$ to disease risk. Hum Mol Genet. 2005;14:3227-3236.

11. Dewan A, Liu M, Hartman S, et al. HTRA1 promoter polymorphism in wet age-related macular degeneration. Science. 2006;314:989-992.

12. Yang Z, Camp NJ, Sun H, et al. A variant of the HTRA1 gene increases susceptibility to age-related macular degeneration. Science. 2006;314: 992-993.

13. Swaroop A, Branham KE, Chen W, Abecasis G. Genetic susceptibility to age-related macular degeneration: a paradigm for dissecting complex disease traits. Hum Mol Genet. 2007;16:R174-R182.

Clinical Ophthalmology

\section{Publish your work in this journal}

Clinical Ophthalmology is an international, peer-reviewed journal covering all subspecialties within ophthalmology. Key topics include: Optometry; Visual science; Pharmacology and drug therapy in eye diseases; Basic Sciences; Primary and Secondary eye care; Patient Safety and Quality of Care Improvements. This journal is indexed on Submit your manuscript here: http://www.dovepress.com/clinical-ophthalmology-journal
14. Gotoh N, Yamada R, Nakanishi H, et al. Correlation between CFHY402H and HTRA1 rs11200638 genotype to typical exudative age-related macular degeneration and polypoidal choroidal vasculopathy phenotype in the Japanese population. Clin Experiment Ophthalmol. 2008;36: 437-442.

15. Gotoh N, Nakanishi H, Hayashi H, et al. ARMS2 (LOC387715) variants in Japanese patients with exudative age-related macular degeneration and polypoidal choroidal vasculopathy. Am J Ophthalmol. 2009;147: 1037-1041.

16. Kondo N, Honda S, Kuno S, et al. Coding variant I62V in the complement factor $\mathrm{H}$ gene is strongly associated with polypoidal choroidal vasculopathy. Ophthalmology. 2009;116:304-310.

17. Sakurada Y, Kubota T, Mabuchi F, et al. Association of LOC387715 A69S with vitreous hemorrhage in polypoidal choroidal vasculopathy. Am J Ophthalmol. 2008;145:1058-1062.

18. Klein MI, Mauldin WM, Stoumbos VD. Heredity and age-related macular degeneration: observation in monozygotic twins. Arch Ophthalmol. 1994;112:932-937.

19. Seddon JM, Cote J, Page WF, et al. The US twin study of age-related macular degeneration: relative roles of genetic and environmental influences. Arch Ophthalmol. 2005;123:321-327.

20. Sho K, Takahashi K, Yamada H, et al. Polypoidal choroidal vasculopathy: incidence, demographic features, and clinical characteristics. Arch Ophthalmol. 2003;121:1392-1396.

21. Maruko I, Iida T, Saito M, et al. Clinical characteristics of exudative agerelated macular degeneration in Japanese patients. Am J Ophthalmol. 2007;144:15-22.

22. Byeon $\mathrm{SH}$, Lee SC, Oh HS, et al. Incidence and clinical patterns of polypoidal choroidal vasculopathy in Korean patients. Jpn $J$ Ophthalmol. 2008;52:57-62.

23. Japanese Study Group of Polypoidal Choroidal Vasculopathy. Criteria for diagnosis of polypoidal choroidal vasculopathy. Nippon Ganka Gakkai Zasshi. 2005;109:417-427.

24. Seddon JM, Hankinson S, Speizer F, Willet WC. A prospective study of cigarette smoking and age-related macular degeneration in women. JAMA. 1996;276:1141-1146.

25. Sperduto RD, Hiller R. Systemic hypertension and age-related maculopathy in the Framingham Study. Arch Ophthalmol. 1986;104: 216-219.

26. Seddon JM, Cote J, Davis N, Rosner B. Progression of age-related macular degeneration: association with body mass index, waist circumference and waist-hip ratio. Arch Ophthalmol. 2003;121:785-792.

27. Rodriguez de Cordoba S, Esparza-Gordillo J, Goicoechea de Jorga E, et al. The human complement factor $\mathrm{H}$ : functional roles, generic variations and disease associations. Mol Immunol. 2004;41:355-367.

28. Penfold PL, Madigan MC, Gillies MC, Provis JM. Immunological and aetiological aspects of macular degeneration. Prog Retin Eye Res. 2001;20:385-414.

29. Kanda A, Chen W, Othman M, et al. A variant of mitochondrial protein LOC387715/ARMS2, not HRTA1, is strongly associated with agerelated macular degeneration. Proc Natl Acad Sci U S A. 2007;104: $16227-16232$.

30. Sakurada Y, Kubota T, Imasawa M, et al. Angiographic lesion size associated with LOC387715 A69S genotype in subfoveal polypoidal choroidal vasculopathy. Retina. 2009;29:1522-1526.

\section{Dovepress}

PubMed Central and CAS, and is the official journal of The Society of Clinical Ophthalmology (SCO). The manuscript management system is completely online and includes a very quick and fair peer-review system, which is all easy to use. Visit http://www.dovepress.com/ testimonials.php to read real quotes from published authors. 